\title{
Simple methods for improving the value of oximetry in the study of pulmonary oxygen uptake
}

\author{
S. L A L , ${ }^{1}$ T. GEBBIE, ${ }^{2}$ AND E. J . M. C A M P B ELL
}

From the Department of Medicine, Postgraduate Medical School, Hammersmith Hospital, London, W.12

The purpose of this paper is to show how an ear oximeter can be used to measure the oxygenation of the blood by the lungs with much greater discrimination than is generally appreciated.

There are three stages: the first is to calibrate the oximeter by a practicable method; the second is to use the instrument to construct an oxygen dissociation curve; and the third is to minimize an important source of variability (the oxygen capacity of the blood) by dealing with differences in saturation instead of absolute saturation.

In normal subjects at sea level the arterial $\mathrm{O}_{2}$ saturation is in the range 92 to $98 \%$. Four sources of variability contribute to this wide range of arterial saturation and its consequent lack of discrimination as a means of assessing respiratory function.

First there is variability in the $\mathrm{Po}_{2}$ of the alveolar air $\left(\mathrm{PAO}_{2}\right)$. This is due chiefly to variation in alveolar ventilation but also to variation in barometric pressure and in the respiratory exchange ratio or respiratory quotient.

Secondly, there is variability in the difference or gradient of $\mathrm{O}_{2}$ pressure between the alveolar air and the arterial blood. This is chiefly due to variation in ventilation: perfusion relationships but also to diffusion, chemical reactions, and 'true' shunts.

Thirdly, there is variability in the relation between arterial $\mathrm{Po}_{2}$ and saturation-that is, variation in the position and slope of the $\mathrm{O}_{2}$ dissociation curve.

Fourthly, there is analytical variability. Some of this is, of course, random, but much of it is systematic and stems from variability in the estimate of the $\mathrm{O}_{2}$ 'capacity' of the blood which causes error because the $\mathrm{O}_{2}$ saturation is $\left(\mathrm{O}_{2}\right.$ content $\div \mathrm{O}_{2}$ capacity) $\times 100$.

Of these four sources of variability the first can now be readily eliminated by estimating arterial

1Present address : Bury General Hospital, Bury, Lancs

'Present address: Wellington Public Hospital, Wellington, New Zealand
$\mathrm{PCO}_{2}$, from which alveolar $\mathrm{Po}_{2}$ can be obtainedo by the use of the alveolar air equation. The third? and fourth are of little interest to respiratory $\vec{c}$ medicine; this leaves the second, which is of great? interest. The standard way of studying this particular aspect of pulmonary gas exchange to-day is $<$ to measure the alveolar-arterial $\mathbf{P o}_{2}$ difference and 0 to calculate the 'venous admixture' or 'shunt' which is equivalent to it. This procedure has limi tations. It requires arterial puncture; continuouso recording is not yet practicable, which makes ob-servation difficult over long periods or duringa changing states such as exercise; and the tech $\frac{\mathrm{O}}{\mathrm{D}}$ niques involve more than a modest outlay and facilities to maintain them in the requisite state of accuracy. We felt that much more use could be made of ear oximetry were the first source of variability (the alveolar $\mathrm{Po}_{2}$ ) eliminated by using. the rebreathing method and alveolar air equation, and if some simple manœuvre were used to eliminate or minimize those in the third and fourth categories.

We shall describe how the desaturation pro duced by rebreathing expired air from a small bag can be used to calibrate an oximeter and how the position and variability of the upper part of the? $\mathrm{O}_{2}$ dissociation curve applicable to the instrument can be determined in normal subjects. This curvebypasses systematic variability between instru- N ments and techniques in the estimate of $\mathbf{O}_{2 N}$ capacity and shows the range of random variation which must be tolerated. As will emerge, this is ${ }^{\omega}$ very much less than expected.

\section{PRELIMINARY CONSIDERATIONS}

The use of certain terms and relationships must be defined.

alveolar $\mathrm{O}_{2}$ PRessure $\left(\mathrm{PAO}_{2}\right)$ This is used to mean the 'ideal' alveolar $\mathrm{Po}_{2}$ calculated from the alveolar air equation, assuming that the arterialo 
$\mathrm{PCO}_{2}\left(\mathrm{PaCO}_{2}\right)$ equals the alveolar $\mathrm{PCO}_{2}$, that is assuming complete equilibration of $\mathrm{CO}_{2}$ between alveolar air and arterial blood.

'END CAPIllaRY' $\mathrm{PO}_{2}\left(\mathrm{PC}^{\prime} \mathrm{O}_{2}\right)$ This is the $\mathrm{Po}_{2}$ of the blood leaving the 'ideal' capillary. The $\mathrm{PO}_{2}$ difference between alveolar air and the endcapillary blood is called the 'end-gradient' and reflects the effects of diffusion and chemical events within the red cell.

ARTerial $\mathrm{PO}_{2}\left(\mathrm{PaO}_{2}\right)$ This is the $\mathrm{PO}_{2}$ of the blood in the systemic arteries. The $\mathrm{Po}_{2}$ difference between the end-capillary and the arterial blood in normal subjects and patients with pulmonary disease is chiefly due to imbalance of ventilation: blood flow relationships and, to a lesser extent, to 'true' anatomical shunts.

BLOOD $\mathrm{O}_{2}$ CONTENT This is the actual volume of $\mathrm{O}_{2}$ carried per unit volume of blood, usually expressed in $\mathrm{ml} . / 100 \mathrm{ml}$.

BLOOD $\mathrm{O}_{2}$ CAPACITY This is the volume of $\mathrm{O}_{2}$ per unit volume of blood when all the haemoglobin is combined with $\mathrm{O}_{2}$.

BLOOD $\mathrm{O}_{2}$ SATURATION Classically this is the blood $\mathrm{O}_{2}$ content expressed as a percentage of the capacity, but there are technical and conceptual difficulties in applying this definition when the highest standards of accuracy are required (Roughton, Darling, and Root, 1944 ; Lundgren, 1961), and in routine use these difficulties cause an uncertainty of up to $\pm 3 \%$ in estimates of 'absolute' or 'true' saturation by volumetric techniques. Spectrophotometric methods measure saturation by determining how much of the haemoglobin is in the oxygenated and how much in the unoxygenated form. It is usual to calibrate these methods for 'true' saturation by reference to a volumetric method, but, as this paper will show, this is unnecessary for the evaluation of respiratory function. It is more valuable to know the slope of the relationship of change in $\mathrm{O}_{2}$ saturation to $\mathrm{O}_{2}$ pressure (i.e., the slope of the dissociation curve) than the relationship of the true saturation to the $\mathrm{O}_{2}$ pressure (i.e., the level of the dissociation curve).

CALIBRATION BY REBREATHING EXPIRED AIR A universally used, rough and ready test for an ear oximeter is to hold the breath and see if the recorded saturation falls. The first procedure described in this paper shows how this manœuvre can be developed into quite a precise technique of calibration by rebreathing from a small bag of expired air. Rebreathing not only prolongs the period of observation beyond that which can be tolerated during simple breath-holding but also provides in the bag the partial pressure of the gases in the lung with which the blood is equilibrated. A large change in both $\mathrm{Po}_{2}$ and saturation can be produced so that several sources of variability become unimportant. This large change is compared with that which would be predicted from a standard dissociation curve and the changes in blood $\mathrm{Po}_{2}$ as revealed by analysis of the air in the bag with which the alveolar air and pulmonary capillary blood come into equilibrium.

The changes in alveolar and blood gases during rebreathing are complex, but four points must be made; first, in normal subjects the deep breaths and small inspired-expired concentration differences mean that the composition of the gas in the bag, the inspired air, expired air, and alveolar air all come to be very similar; secondly, in normal subjects the difference in alveolar $\mathrm{Po}_{2}$ and arterial $\mathrm{Po}_{2}$ due to the processes of $\mathrm{O}_{2}$ uptake and ventilation:perfusion imbalance are unimportant ${ }^{1}$; thirdly, the range of $\mathrm{PCO}_{2}, p \mathrm{H}$, and saturation in normal subjects breathing air is so small that, provided a big enough change is produced, average normal values can be safely assumed for the start of the rebreathing procedure; fourthly, the change in blood $\left(\mathrm{H}^{+}\right)$ or $\mathrm{pH}$ can be estimated from the change in $\mathrm{PCO}_{2}$ with sufficient accuracy.

DETERMINATION OF UPPER PART OF DISSOCIATION CURVE The second procedure makes use of the known relationships between alveolar and endcapillary $\mathrm{Po}_{2}$ and between the $\mathrm{O}_{2}$ saturation of end-capillary and arterial blood in normal subjects.

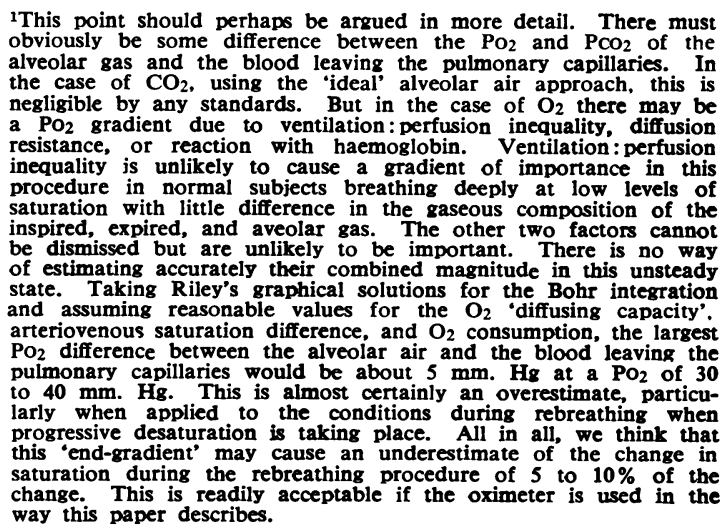

1This point should perhaps be argued in more detail. There must alveolar gas and the blood leaving the pulmonary capillaries. In the case of $\mathrm{CO}_{2}$, using the 'ideal' alveolar air approach, this is negligible by any standards. But in the case of $\mathrm{O}_{2}$ there may be a $\mathrm{PO}_{2}$ gradient due to ventilation: perfusion inequality, diffusion resistance, or reaction with haemoglobin. Ventilation:perfusion procedure in normal subjects breathing deeply at low levels of saturation with little difference in the gaseous composition of the inspired, expired, and aveolar gas. The other two factors cannot be dismissed but are unlikely to be important. There is no way
of estimating accurately their combined magnitude in this unsteady state. Taking Riley's graphical solutions for the Bohr integration and assuming reasonable values for the $\mathrm{O}_{2}$ 'diffusing capacity'. arteriovenous saturation difference, and $\mathrm{O}_{2}$ consumption, the largest pulmonary capillaries would be about $5 \mathrm{~mm}$. $\mathrm{Hg}$ at a $\mathrm{PO}_{2}$ of 30 to $40 \mathrm{~mm}$. Hg. This is almost certainly an overestimate, particularly when applied to the conditions during rebreathing when
progressive desaturation is taking place. All in all, we think that this 'end-gradient' may cause an underestimate of the change in saturation during the rebreathing procedure of 5 to $10 \%$ of the
change. This is readily acceptable if the oximeter is used in the way this paper describes. 
There are two steps in the reasoning. First, at $\mathrm{Po}_{2}$ s over $60 \mathrm{~mm}$. $\mathrm{Hg}$ alveolar $\mathrm{Po}_{2}$ is virtually the same as end-capillary $\mathrm{Po}_{2}$, and both can be varied to a known degree by changing the inspired $\mathrm{O}_{2}$ concentration; secondly, the end-capillary $\mathrm{O}_{2}$ saturation is about $1 \%$ greater than the arterial (range 0.5 to $1.5 \%$ ). This estimate is arrived at as follows: in a normal subject the proportion of wholly or partially shunted blood in the systemic arteries is less than $5 \%$ of the total cardiac output (Farhi and Rahn, 1955). The reduction in systemic arterial saturation $\left(\mathrm{SaO}_{2}\right)$ at rest due to a $5 \%$ shunt (assuming an a-v difference of $30 \%$ saturation at rest) would be $1.5 \%$ (i.e., $\frac{5}{1} \frac{5}{0} \times 30=1 \cdot 5$ ). The reduction is usually 0.75 to $1.0 \%$. We can therefore take $\mathrm{Sc}^{\prime} \mathrm{O}_{2}$ as lying between $\left(\mathrm{SaO}_{2}+0 \cdot 75\right)$ and $\left(\mathrm{SaO}_{2}+1 \cdot 5\right)$, say $\left(\mathrm{SaO}_{2}+1\right)$.

If $\mathrm{Sc}^{\prime} \mathrm{O}_{2}$ is plotted against $\mathrm{Pc}^{\prime} \mathrm{O}_{2}$, a dissociation curve is obtained. We determined the $\mathrm{PAO}_{2}$ $\left(\simeq \mathrm{Pc}^{\prime} \mathrm{O}_{2}\right)$ and observed the $\mathrm{SaO}_{2}\left(\simeq \mathrm{Sc}^{\prime} \mathrm{O}_{2}-1\right)$ in normal subjects at rest both while breathing air $\left(\mathrm{FIO}_{2} \mathbf{0}^{2093}\right)$ and a low $\mathrm{O}_{2}$ mixture $\left(\mathrm{FIO}_{2} \mathbf{0} \cdot 1599\right.$ to $0 \cdot 1855$ ). Thus we obtained readings over a wide range of the upper part of the $\mathrm{O}_{2}$ dissociation curve.

\section{APPARATUS AND SUBJECTS}

ATLAS OXIMETER TYPE E.M. 45B The oximeter was allowed to warm up for one hour before use. It was set up according to the maker's instructions. On first using the instrument this procedure seemed to be rather laborious, but with practice it could be carried out within 15 minutes. Three gain settings were used (Fig. 2).

REBREATHING BAG This was of volume 31 . with sampling leads at the neck.

GAS ANALYSER A Lloyd-Haldane (Lloyd, 1958) or Scholander (1947) gas analyser was used́.

MASS SPECTROMETER TYPE MS4 A.E.I.

RAPID $\mathrm{CO}_{2}$ ANALYSER Infra Red Company Type R.M.

We found it convenient to use the mass spectrometer and the Infra Red analyser, but they are not essential for the procedure (see Discussion).

The subjects used in the study were normal healthy adults with no history of cardiovascular or respiratory disease, normal chest radiographs, normal exercise tolerance, and normal F.E.V.1.0 and vital capacity.

\section{CALIBRATION BY REBREATHING}

The protocol was as follows:

(1) The oximeter was set to read $100 \%$ saturation after the subject had been breathing $100 \% \mathrm{O}_{2}$ for sufficient time for the reading to become constant. This usually took about 5 minutes.

(2) The reading for saturation was noted after the $\mathrm{O}_{2}$ had been washed out of the lungs by breathing air for 10 to 15 minutes.

(3) The subject rebreathed his expired gas from a bag of $3 \mathrm{l}$. volume for as long as he could tolerate, $\vec{P}$ or until the saturation fell to about $50 \%$, or until. the $\mathrm{Po}_{2}$ fell to about $30 \mathrm{~mm}$. $\mathrm{Hg}$. This usually took $1 \frac{1}{2}$ to 2 minutes.

(4) The lung-to-ear circulation time (about 5 sec.) $\underset{\vec{x}}{\stackrel{\vec{x}}{x}}$ was determined from the delay between the end of $i$ rebreathing and the subsequent rise in saturation. Allowance was made for this delay in the subsequent $\vec{i}$ analyses.

The $\mathrm{O}_{2}$ and $\mathrm{CO}_{2}$ concentrations in the bag at the 을 end of rebreathing were measured with the Scholander $\vec{c}$ or Lloyd-Haldane apparatus. During rebreathing the gas passing back and forth from the bag to the lungs through the mouthpiece was continuously sampled and analysed with the mass spectrometer (Fig. 1). The saturation as shown on the oximeter was recorded repeatedly.

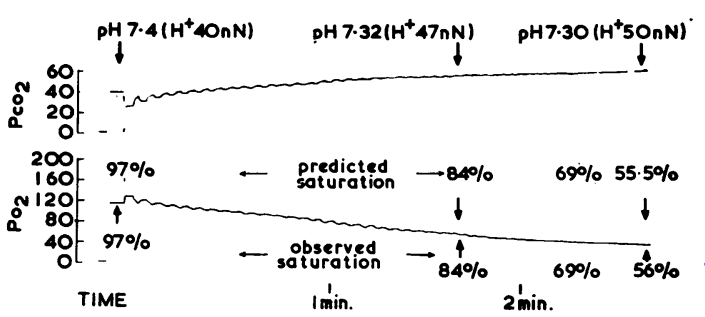

FIG. 1. Mass spectrometer tracings of $\mathrm{PCO}_{2}$ and $\mathrm{Po}_{2}$ sampled continuously at the mouth of $a$ bag from which a normal subject was rebreathing expired air. The values for $\left(\mathrm{H}^{+}\right)$were predicted from the change in $\mathrm{PCO}_{2}$, and the predicted saturation values were obtained from an oxygen dissociation curve. The observed saturation values were those shown by the oximeter, allowance being 옥 made for the circulation time.

The predicted saturation corresponding to each estimate of the alveolar $\mathrm{Po}_{2}$ was obtained from the $\mathrm{Gr}$ standard dissociation curve of Dill (1944). Allow- N ance for the change in blood $p \mathrm{H}$ (or $\mathrm{H}^{+}$activity) $\mathrm{N}_{\mathrm{N}}$ was made as follows. A normal $\left(\mathrm{H}^{+}\right)$(40 nano- $O$ Normal) and $\mathrm{PCO}_{2}(40 \mathrm{~mm}$. $\mathrm{Hg})$ were assumed at calculated from the final $\mathrm{PCO}_{2}$ using a $\mathrm{PCO}_{2} / \mathrm{HCO}_{3}^{-} \stackrel{0}{\complement}$ the start of rebreathing. The final $\left(\mathrm{H}^{+}\right)$was then diagram (Campbell, 1962), assuming that the wholebody dissociation curve resembles that of blood. The dissociation curve for the body is somewhat less steep than that for blood (Brackett, Cohen, and Schwartz, 1965), but the effect of this difference on the calcu- $\mathbb{D}$ lated $\left(\mathrm{H}^{+}\right)$and saturation is unimpcrtant in the range observed. 


\section{DETERMINATION OF UPPER PART OF DISSOCIATION CURVE}

The protocol for this study was:

(1) The oximeter was switched on to warm up for an hour ; the subject rested for 10 to 15 minutes.

(2) The oximeter was set on the ear according to the maker's instructions with an added operating gain of $10 \%$ (see below). The subject breathed $100 \% \mathrm{O}_{2}$ for a few minutes; the oximeter was set at $100 \%$ saturation. The subject breathed air, and the $\mathrm{SaO}_{2}$ was obtained from the oximeter reading after the excess $\mathrm{O}_{2}$ had been washed out from the lungs.

(3) Expired gas was collected for 4 to 5 minutes in a previously flushed Douglas bag. Saturation readings were taken every 15 seconds during the period.

(4) The mixed venous $\mathrm{PCO}_{2}\left(\mathrm{P} \overline{\mathrm{v}} \mathrm{CO}_{2}\right)$-and hence arterial $\mathrm{PCO}_{2}$-was estimated by the two-stage rebreathing method of Campbell and Howell (1960) or the one-stage method of Collier (1956).

(5) The inspired gas mixture was changed from air to the low $\mathrm{O}_{2}$ mixture.

After 10 to 15 minutes of quiet breathing, (3) and (4) were repeated.

(6) The expired gas was analysed for $\mathrm{O}_{2}, \mathrm{CO}_{2}$, and N2.

(7) The barometric pressure and temperature were noted.

Calculation of $\mathrm{Sc}^{\prime} \mathrm{O}_{2}$ and $\mathrm{PC}^{\prime} \mathrm{O}_{2}$ The $\mathrm{Sc}^{\prime} \mathrm{O}_{2}$ was taken to equal $\mathrm{SaO}_{2}+1$ and the $\mathrm{Pc}^{\prime} \mathrm{O}_{2}$ was taken to be equal to $\mathbf{P A O}_{2}$ (see preliminary considerations). $\mathbf{P A O}_{2}$ was calculated from the alveolar air equation, assuming that alveolar $\mathrm{PCO}_{2}$ was equal to arterial $\mathrm{PCO}_{2}$; arterial $\mathrm{PCO}_{2}$ was derived from the measured mixed venous $\mathbf{P C O}_{2}$, assuming an arteriovenous $\mathbf{P C O}_{2}$ difference of $6 \mathrm{~mm}$. Hg (Hackney, Sears, and Collier, 1958; Campbell and Howell, 1960). We used this method to determine the $\mathrm{PAO}_{2}$ (and hence $\mathrm{Pc}^{\prime} \mathrm{O}_{2}$ ) rather than any direct procedure, such as end-tidal sampling, for two reasons: first, because it is easier to use this method than end-tidal sampling in a routine clinical laboratory, and, secondly, end-tidal sampling cannot be used to estimate effective alveolar $\mathrm{Po}_{2}$ in subjects with abnormal ventilation:blood flow relationships.

\section{RESULTS}

REBREATHING CALIBRATION The relationship between observed and predicted changes in saturation were linear at all three gain settings (Fig. 2).

The agreement using the recommended operating gain was fairly good, but the readings slightly under-estimated the predicted changes in saturation. Increasing the gain by $40 \%$ caused the changes to be greatly over-estimated. When the procedure was repeated with an added operating gain of $10 \%$ the agreement was good. We thus

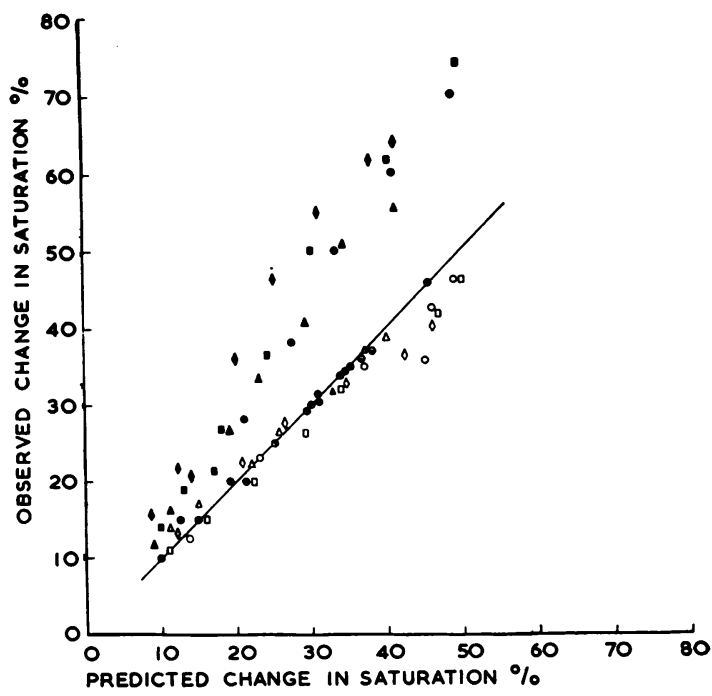

FIG. 2. Observed changes in saturation plotted against the predicted changes. Each symbol represents an individual subject. The open symbols represent findings at the setting of the oximeter recommended by the manufacturers. The solid symbols represent the readings with the operating gain of the oximeter increased by $40 \%$, and the partially filled symbols represent the findings with the operating gain increased by $10 \%$.

concluded that this particular instrument required an operating gain $10 \%$ above that arrived at if the maker's instructions for setting the instrument were followed.

THE DISSOCIATION CURVE The derived values of $\mathrm{Pc}^{\prime} \mathrm{O}_{2}$ and $\mathrm{Sc}^{\prime} \mathrm{O}_{2}$ are plotted in Figure 3. Twentyeight points were obtained. The regression of saturation on pressure is :

$$
\mathrm{Sc}^{\prime} \mathrm{O}_{2}=0 \cdot 111 \mathrm{Pc}^{\prime} \mathrm{O}_{2}+86 \cdot 15 \text {. }
$$

The outer two lines (AB, CD) show the $95 \%$ confidence limits of the observations. It will be seen that saturation may be predicted from $\mathbf{P o}_{2}$ to within $\pm 2 \%$.

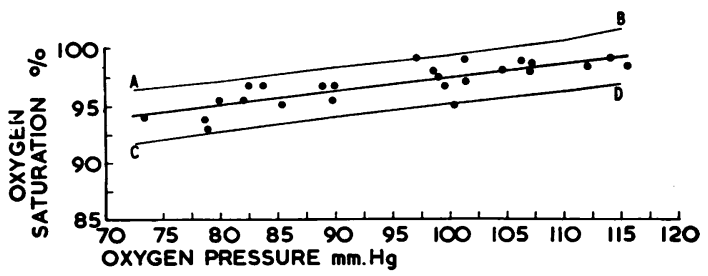

FIG. 3. Upper range of the dissociation curve in 14 subjects. Two readings were obtained for each subject at $\mathrm{FIO}_{2}$ 0.209 and about $0 \cdot 17$. Lines $A B$ and $C D$ are the $95 \%$ confidence limits of the observations. 
The results were examined to see the importance of various factors in the hope that the procedure could be simplified by omitting some of the more tedious measurements. We accordingly assumed a barometric pressure of $760 \mathrm{~mm}$. $\mathrm{Hg}$ and respiratory exchange ratio of 0.8 and repeated the calculations. In doing this we were in some difficulty because the average $\mathbf{R}$ of our subjects while breathing low $\mathrm{O}_{2}$ was unduly high, probably because the period of wash-in had not been long enough to attain a steady state. Despite this the results of simplification were satisfactory (Fig. 4).

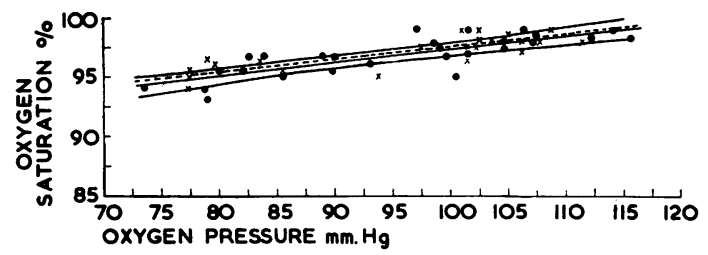

FIG. 4. Effect of simplifying the data on the calculated dissociation curve. The data of Fig. 3 are shown as dots, and the solid line is the regression of these points. The data obtained by assuming a respiratory-exchange ratio of 0.8 and barometric pressure of $760 \mathrm{~mm} . \mathrm{Hg}$ are shown as crosses, and the dotted line is the regression of these points. The curved lines are the confidence limits of the first regression line. The assumptions have not significantly affected the slope.

The second regression line (dotted line) has altered a little but differs in position by no more than $0.6 \%$ saturation over the range. The regression of saturation on pressure is:

$$
\mathrm{Sc}^{\prime} \mathrm{O}_{2}=0.099 \mathrm{Pc}^{\prime} \mathrm{O}_{2}+87.62 \text {. }
$$

The slopes of the two lines are not significantly different. Although the intercepts are significantly different, the difference is unimportant in the upper range of the curve, since the points are completely enclosed by the confidence limits of the first regression line. This difference could probably have been avoided had a more steady state been achieved while breathing the low $\mathrm{O}_{2}$ mixture. Assumption of $\mathbf{R}$ and barometric pressure therefore appears to be justifiable and thus obviates the need for expired gas collection and analysis.

\section{DISCUSSION}

Ear oximetry is a useful and attractive technique since it provides a continuous reading of arterial saturation and removes the need for arterial puncture. The accuracy of the technique is, how $\overrightarrow{\vec{c}}$ ever, generally regarded with some misgiving, if being thought that changes in saturation can be measured more accurately than the absolute values. Nilsson (1960) remarks that in manjo situations an accurate calibration is not importan as, for instance, in studies of the time-course or s relative magnitude of saturation changes and in $\vec{b}$ circulation-time studies. Should absolute satura-tion be required, the usual practice is to check the readings by analysis of drawn blood using methods such as that of Van Slyke and Neill (1924). In other than skilled and practised hands this gesture is more sacramental than scientific Oximeters are also regarded as temperamental ;o Wood, Sutterer, and Cronin (1960) suggest thaf instruments should be individually and repeatedlycalibrated, as they are subject to unpredictablec changes in their characteristics with age and conditions of use.

The use of ear oximetry inevitably introducese analytical errors greater than those experiencedos in standard spectrophotometry because it does not satisfy any of the conditions which should beo present before the Lambert-Beer Law can be applied. The light used is not monochromatic; ito is passed through a particulate suspension (blood) in a variable cuvette (the ear). However, by using the method described the random effects of theseô variables on the performance of the individual ${ }^{3}$ instrument can be assessed and the systematic effects of these and some other physiologicals variables can be eliminated as described in the introduction.

It should be noted that if the oximeter is to be used only for evaluating pulmonary function, the accuracy required of the rebreathing procedureô need not be very great because the dissociation curve determined by the second procedure is stillo usable even if its slope is slightly wrong. Ironically, $>$ the data for directly determined $\mathrm{O}_{2}$ dissociation curves in the usual arterial range in all the papers we examined were much less extensive and more ${ }^{\mathrm{G}}$ variable than those in Figure 3.

We did not attempt to define the $\mathrm{O}_{2}$ dissociation ${ }^{\mathrm{\omega}}$ curve in the region where its slope is changing? rapidly or in its lower, steep part for two reasons : first, it is technically more difficult and some of $\stackrel{\mathscr{D}}{\mathbb{D}}$ the assumptions become less justifiable ; secondly, the aim of the study was to improve the value of oximetry in detecting minor degrees of $\stackrel{\mathbb{D}}{\circ}$ unsaturation, its accuracy in recording more gross $\frac{\widetilde{d}}{\mathrm{D}}$ changes being sufficiently revealed by the rebreath- $\alpha$ ing procedure. 
TABLE I

COMPARISON OF VENOUS ADMIXTURE AS ESTIMATED BY OXIMETRY USING THE PROCEDURE DESCRIBED AND AS ESTIMATED BY ALVEOLAR-ARTERIAL (A-a) PO, DIFFERENCE MEASURED BY ELECTRODES

\begin{tabular}{|c|c|c|c|c|}
\hline \multirow{2}{*}{$\begin{array}{l}\text { Sub- } \\
\text { ject }\end{array}$} & \multirow{2}{*}{ Diagnosis } & \multirow{2}{*}{$\begin{array}{l}\text { Predicted- } \\
\text { observed } \\
\text { Saturation } \\
\text { (oximetry) }\end{array}$} & \multicolumn{2}{|c|}{$\begin{array}{c}\text { Equivalent R-L } \\
\text { Shunt (\% of Systemic } \\
\text { Blood Flow) } \\
\end{array}$} \\
\hline & & & $\begin{array}{c}\text { By } \\
\text { Oximetry }\end{array}$ & $\begin{array}{c}\text { By } \mathbf{A}-\mathbf{a} \\
\text { Difference }\end{array}$ \\
\hline $\begin{array}{l}\text { E.W. } \\
\text { L.M. }\end{array}$ & $\begin{array}{l}\text { Bronchiectasis; diffuse } \\
\text { airways obstruction } \\
\text { Pulmonary fibrosis; }\end{array}$ & $5 \cdot 5$ & 18 & 25 \\
\hline J.K. & $\begin{array}{l}\text { portal hypertension } \\
\text { Sarcoidosis } \\
\text { Chronic bronchitis ; }\end{array}$ & $\begin{array}{l}4 \cdot 5 \\
1 \cdot 5\end{array}$ & $\begin{array}{r}15 \\
5\end{array}$ & $\begin{array}{r}14 \\
6\end{array}$ \\
\hline $\begin{array}{l}\text { D.B. } \\
\text { A.W. } \\
\text { E.M. } \\
\text { C.P. }\end{array}$ & $\begin{array}{l}\text { alveolar hypoventila- } \\
\text { tion } \\
\text { Pulmonary fibrosis } \\
\text { Asbestosis } \\
\text { Chronic bronchitis } \\
\text { Chronic bronchitis }\end{array}$ & $\begin{array}{l}3 \cdot 5 \\
4 \cdot 5 \\
3 \\
4 \cdot 5 \\
4\end{array}$ & $\begin{array}{l}11 \\
15 \\
10 \\
15 \\
14\end{array}$ & $\begin{array}{r}7 \\
20 \\
10 \\
20 \\
18\end{array}$ \\
\hline
\end{tabular}

Table I gives a comparison of the venous admixture effect estimated by this usage of oximetry with the standard procedure based on direct arterial $\mathrm{Po}_{2}$ measurement in patients with minor degrees of ventilation:perfusion inequality. The agreement is very satisfactory and probably within the limits of accuracy of either method.

RECOMMENDED PROCEDURE (1) Set the oximeter on the ear of a normal subject. Have him breathe $100 \%$ oxygen until the reading is constant for one minute. Set saturation to read $100 \%$. Allow the excess $\mathrm{O}_{2}$ to be washed out of the lungs by breathing air. Note the saturation. Have the subject rebreathe expired air from a 3-litre bag until the saturation falls to about $50 \%$ or until the limit of tolerance of the subject is reached. If the instrument is defective some judgment may be needed here to prevent the subject from becoming too hypoxic. Usually, however, the dyspnoea produced by the combination of hypoxia and $\mathrm{CO}_{2}$ accumulation limits the procedure. Since this study was completed we have obtained a paramagnetic $\mathbf{O}_{2}$ analyser (Servomex) which is inexpensive, reliable, and sufficiently rapid in response to give the $\mathrm{O}_{2}$ concentration in the bag within 10 to 20 seconds. We therefore now use this analyser to sample the bag continuously and carry on the rebreathing until the oxygen concentration falls to 4.5 to $5 \%$ $\left(\mathrm{Po}_{2}=30\right.$ to $35 \mathrm{~mm}$. $\left.\mathrm{Hg}\right)$. Note the lowest saturation reached. Measure the $\mathrm{O}_{2}$ and $\mathrm{CO}_{2}$ percentage in the bag and obtain the predicted saturation as described in detail on p. 52. Plot the observed change in saturation against the predicted change. Either use this as a calibration or adjust the instrument and repeat.
(2) A dissociation curve should be obtained for an adequate sample of the normal population ideally by breathing air and an $\mathrm{O}_{2}$ in $\mathrm{N}_{2}$ mixture of about 16 to $17 \%$. If a long enough period of breathing is allowed (say 20 to 30 minutes) an $\mathbf{R}$ of 0.8 may be assumed. Plot arterial saturation +1 against calculated alveolar $\mathrm{Po}_{2}$.

If procedure (1) is used to check the instrument from time to time, procedure (2) need not be repeated.

(3) When studying a patient the oximeter should be set to read a lower than probable value while breathing air, say $80 \%$. (Adjusting the reading to $100 \%$ while breathing $\mathrm{O}_{2}$ is not recommended, partly to save time but chiefly because there may be no scale reading above $100 \%$ which can be used if, with the passage of time during an experiment, the instrument comes to 'read higher'.) The two-stage rebreathing procedure for the estimation of mixed venous $\mathrm{PCO}_{2}$ should then be carried out. During the first stage-that is, while rebreathing a high $\mathrm{O}_{2}$ mixture - the rise in saturation $\left(\triangle \mathrm{SaO}_{2}\right)$ should be noted. If the saturation has not finished rising before the end of this procedure the subject should be given $\mathrm{O}_{2}$ to breathe until it has finished rising. Observed arterial saturation is then $100-\triangle \mathrm{SaO}_{2}$. Predicted pulmonary capillary saturation is estimated by using the operational dissociation curve to find the saturation value corresponding to $\mathrm{PAO}_{2}=150-1 \cdot 2 \quad\left(\mathrm{P}_{\overline{\mathrm{V}} \mathrm{CO}}-6\right)$.

The observed saturation should not be more than $2 \%$ below the predicted. The confidence of the estimated difference between the observed and the predicted saturation is indicated by the variability of the data from which the dissociation curve was derived. If the pulmonary capillaryvenous $\mathrm{O}_{2}$ saturation difference $\mathrm{S}(\mathrm{c}-\overline{\mathrm{v}})$ is known or can be assumed, the difference between the observed and predicted saturation $\left(\triangle \mathrm{SaO}_{2}\right)$ can be expressed as the equivalent of a shunt equal to $\frac{\triangle \mathrm{SaO}_{2}}{\mathrm{~S}(\mathrm{c}-\overline{\mathrm{v}})} \times 100 \%$ of systemic arterial blood flow.

\section{SUMMARY}

Two complementary methods of calibrating an ear oximeter have been examined. Both use the lungs of normal subjects as a tonometer and obviate the need for arterial blood sampling.

(1) The accuracy in measuring change in saturation was estimated by having a normal subject rebreathe from a bag of expired air producing equilibrium between the bag, the alveolar gas, and 
the pulmonary capillary blood. The $\mathrm{O}_{2}$ and $\mathrm{CO}_{2}$ concentrations in the bag were measured and saturation was predicted from a standard dissociation curve taking into account the change in the hydrogen ion activity. This saturation was compared with the observed saturation on the oximeter and a calibration curve was obtained.

(2) A dissociation curve was constructed, using the instrument, by estimating the $\mathrm{O}_{2}$ pressure and saturation in the pulmonary capillary blood $\left(\mathrm{Pc}^{\prime} \mathrm{O}_{2}\right.$ and $\mathrm{Sc}^{\prime} \mathrm{O}_{2}$ ). Alveolar $\mathrm{Po}_{2}$ was estimated from mixed venous $\mathrm{PCO}_{2}$ and the alveolar air equation. $\mathrm{Pc}^{\prime} \mathrm{O}_{2}$ was taken to be the same as alveolar $\mathrm{Po}_{2}$. Saturation at the ear $\left(\mathrm{SaO}_{2}\right)$ was measured by the oximeter and the $\mathrm{Sc}^{\prime} \mathrm{O}_{2}$ was taken to be $\mathrm{SaO}_{2}+1$. The method was further simplified by assuming a barometric pressure of $760 \mathrm{~mm}$. $\mathrm{Hg}$ and a respiratory exchange ratio of 0.8 without clinically significant loss of accuracy.

Simplifications of these methods are described. Provided the first procedure is used occasionally to check the instrument (the time required for this and its simplicity facilitate frequent calibration), the second procedure need only be carried out once.

If the upper part of the dissociation curve is obtained by the method described, several sources of variability in the measurement of arterial saturation can be greatly reduced and the detection of arterial unsaturation due to defective pulmonary uptake can be improved.
Some of the work reported forms part of a thesis submitted by S. L. for the M.D. of Punjab Universitye We are indebted to Dr. N. L. Jones for help with some of the experiments, to Miss H. M. Ferres, of the R.A.F. Institute of Aviation Medicine, Farn@ borough, for help with the statistics, to Dr. T. J. $\mathrm{H}_{\infty}^{0}$ Clark, Dr. G. J. R. McHardy, and Dr. J. B. West for criticism of the manuscript, and to Mrs. E. Wines for the illustrations.

\section{REFERENCES}

Brackett, N. C., Cohen, J. J., and Schwartz, W. B. (1965). Carbo采 dioxide titration curve of normal man. Effect of increasing degrees of acute hypercapnia on acid-base equilibrium. New Engl.J. Med., 272, 6.

Campbell, E. J. M. (1962). RIpH. Lancet, 1, 681 .

and Howell, J. B. L. (1960). Simple rapid methods of estimating arterial and mixed venous pCO $\mathrm{CO}_{2}$ Brit.med. J., 1, 458.

Collier, C. R. (1956). Determination of mixed venous $\mathrm{CO}_{2}$ tensions by rebreathing. J. appl. Physiol., 9, 25.

Dill, D. B. (1944). Handbook of Respiratory Data in Aviation. National Research Council, Washington.

Farhi, L. E., and Rahn, H. (1955). A theoretical analysis of th alveolar-arterial $\mathrm{O}_{2}$ difference with special reference to the distribution effect. J. appl. Physiol., 7, 699.

Hackney, J. D., Sears, C. H., and Collier, C. R. (1958). Estimation of arterial $\mathrm{CO}_{2}$ tension by rebreathing technique. Ibid., 12, 425 . Lloyd, B. B. (1958). A development of Haldane's gas-analysis appa
ratus. J. Physiol. (Lond.), 143, 5P.

Lundgren, C. E. G. (1961). Oxygen dissociation curve of huma@ blood equilibrated with high concentrations of oxygen. Scanto J. clin. Lab. Invest., 13, 291.

Nilsson, N. J. (1960). Oximetry. Physiol. Rev., 40, 1.

Roughton, F. J. W., Darling, R. C., and Root, W. S. (1944). Factor affecting the determination of oxygen capacity, content and pressure in human arterial blood. Amer. J. Physiol., 142, 708.

Scholander, P. F. (1947). Analyzer for accurate estimation of re spiratory gases in one-half cubic centimeter samples. J. bio Chem., 167, 235.

Van Slyke, D. D., and Neill, J. M. (1924). The determination of gase in blood and other solutions by vacuum extraction and manometric measurement. Ibid., 61, 523.

Wood, E. H., Sutterer, W. F., and Cronin, L. (1960). Oximetry. ছি Medical Phvsics, ed. O. Glasser, Vol. 3, p. 416. Year Book Publishers, Chicago. 